\title{
PBAR Note 631 \\ Dimensions and Measurements of Debuncher Band 1 and 2 Waveguide-Coax Launchers (Final Version)
}

\section{Ding Sun \\ $02 / 15 / 2000$}

This note is a document about dimensions and measurement results of waveguidecoax launchers (Band 1 and 2) installed on the arrays in debuncher cooling upgrade.

Shown in Figure 1, 5, 8 and 12 are schematic drawings of launchers in the cross section along the longitudinal direction (beam direction) of the arrays. The unit in these drawings is inch. Note: although there are upper band and lower band for pickup arrays, the launchers are the same to avoid possible confusion during installation.

RF Measurements were made on all launchers (port) and printed in hard copies for future reference. Since the measurement results are similar to each other, only a few plots for each type of launcher/band are presented in this document.

There are two types of measured S11 parameters. One is the measurement made at the end of design/tuning stage using a straight section of band 1 or 2 waveguide terminated with a cone of absorber. I use "Original" to denote this kind of measurement. As shown in Figure 2, 6, 9 and 13, the original S11 of all launchers are below or around $20 \mathrm{db}$ over the full band 1 or 2 .

The other type of measurement is the one made after these launchers were installed onto the array including elbows and several type $\mathrm{N}$ feedthrough or connectors. The kicker arrays were terminated with wedges of absorber. During all measurements (pickup array or kicker array) when one launcher was being measured, all other launchers were terminated with 50 ohm terminator. As shown in Figure 3, 4, 7, 10, 11 and 14 these "final" S11s are around $-15 \mathrm{db}$.

Note: shown in Figure 7 is the S11 result of Band 1 horizontal prototype kicker. This Kicker is replaced by a new one. The S11 of new band 1 kicker is better than the one in Figure 7 (available only in hard copy).

Note: the rule for denoting the port number is as follows. From the upper stream to the down stream of the tank, the port number on one side of the tank (for example on the wall side) is $1,3,5$ and 7 while on the other side of the tank (for example on the aisle side) is $2,4,6$ and 8 . 


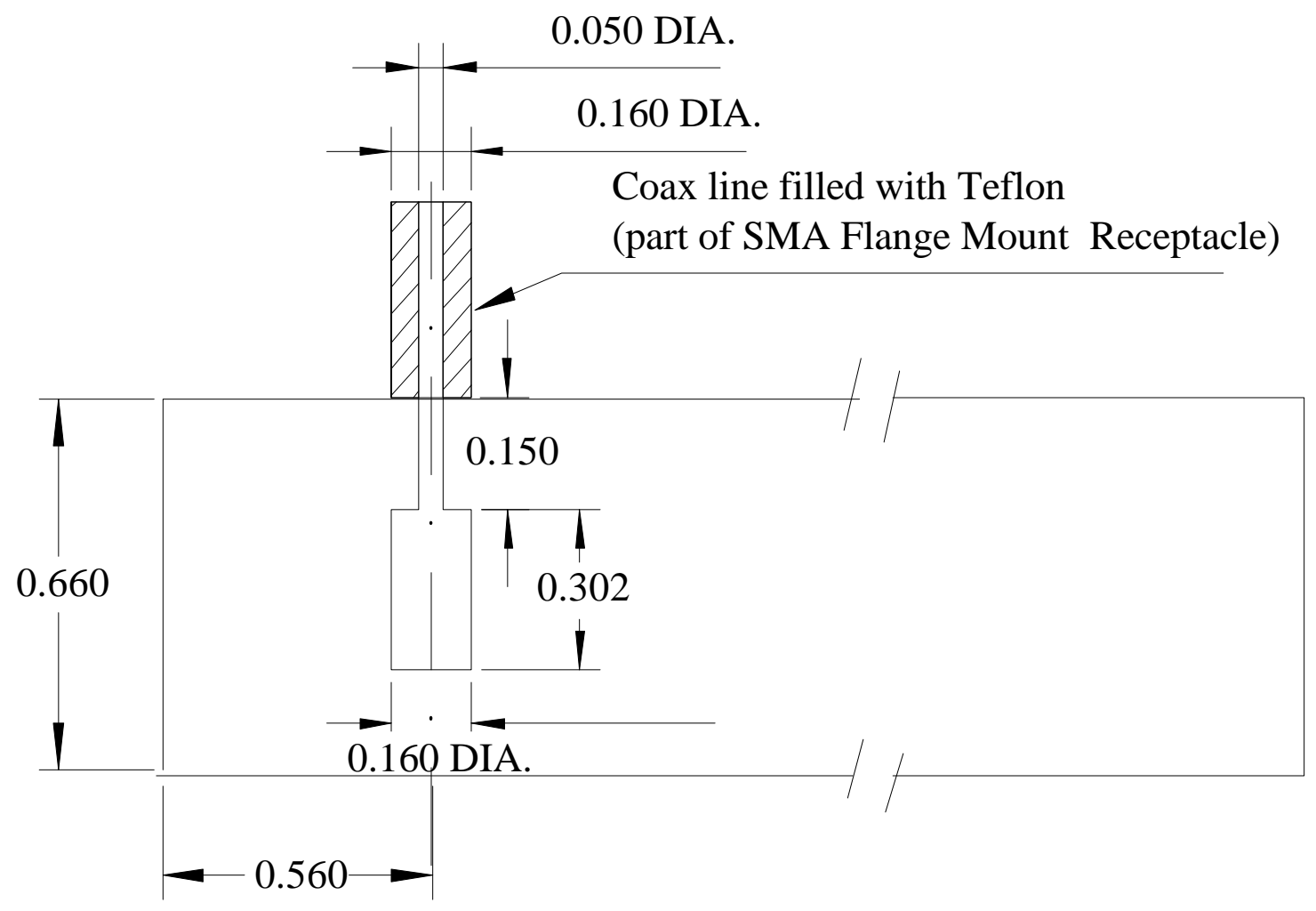

Longitudinal Direction

Figure 1. Dimension of WG-Coax Launcher (Band 1 Pickup) 


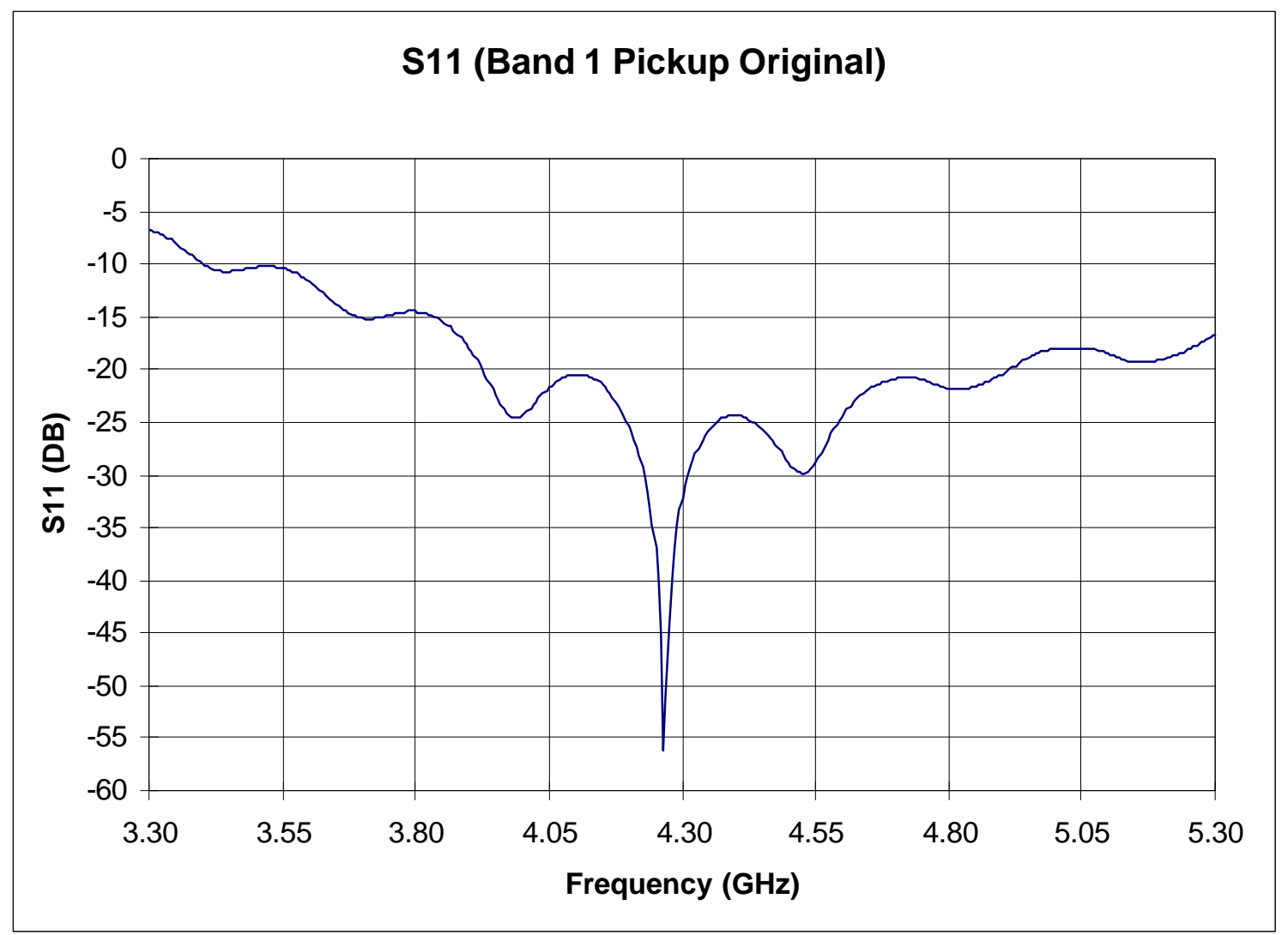

Figure 2. S11 of WG-Coax Launcher (Band 1, Pickup, measured using test setup- a straight section of waveguide) 


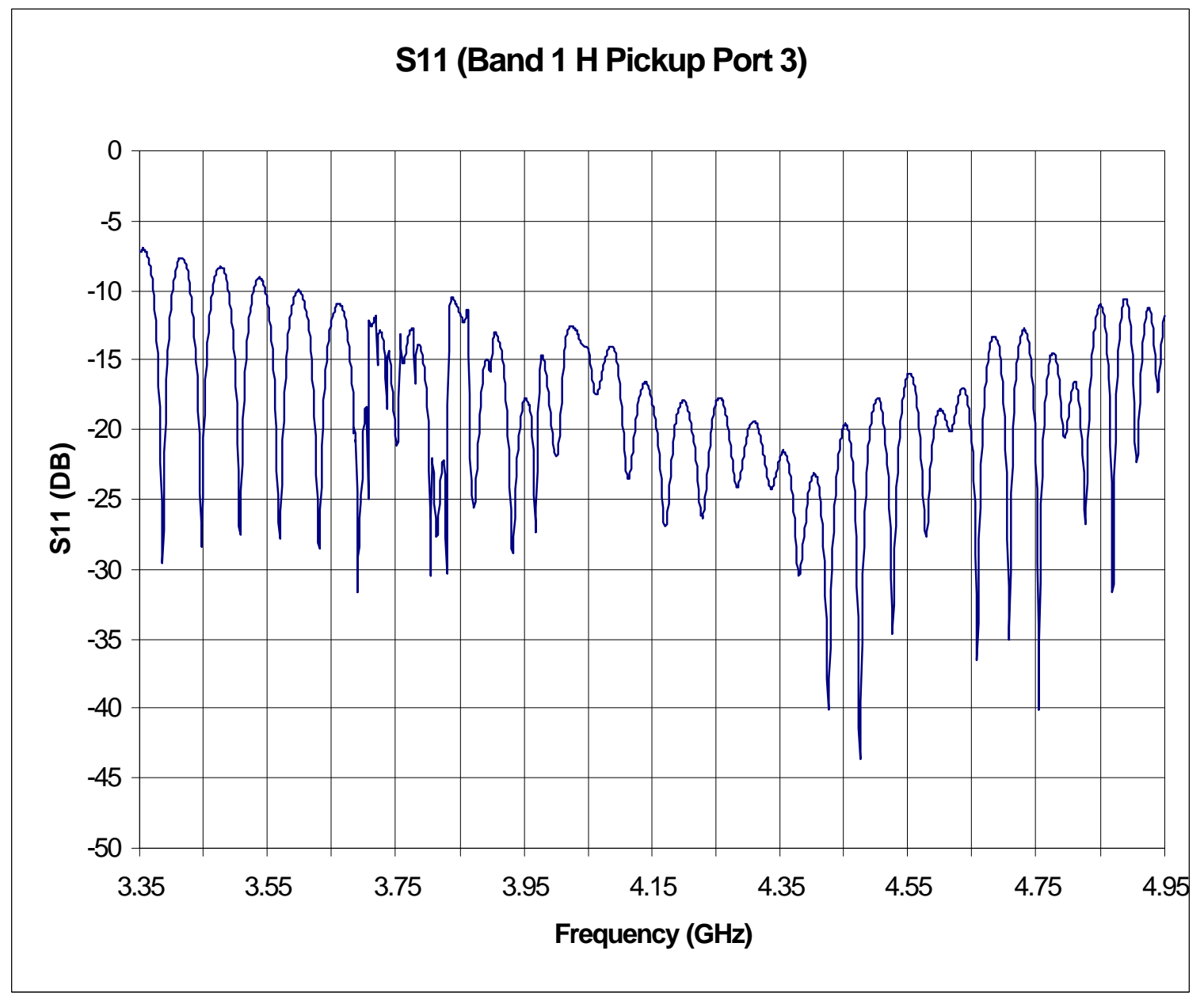

Figure 3. S11 of WG-Coax Launcher (Band 1, Pickup, upper stream, measured after being installed onto array) 


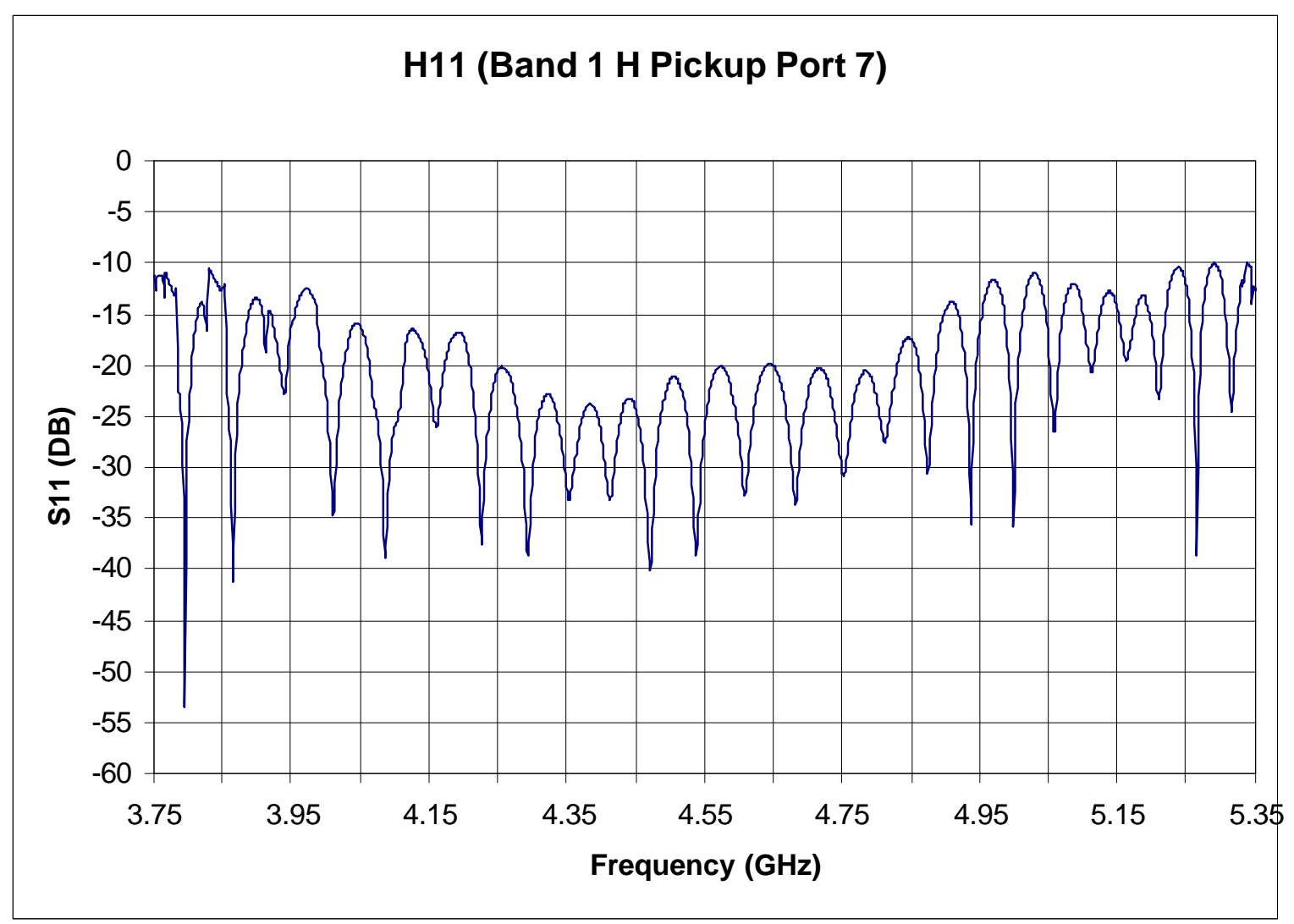

Figure 4. S11 of WG-Coax Launcher (Band 1, Pickup, down stream, measured after being installed onto array) 


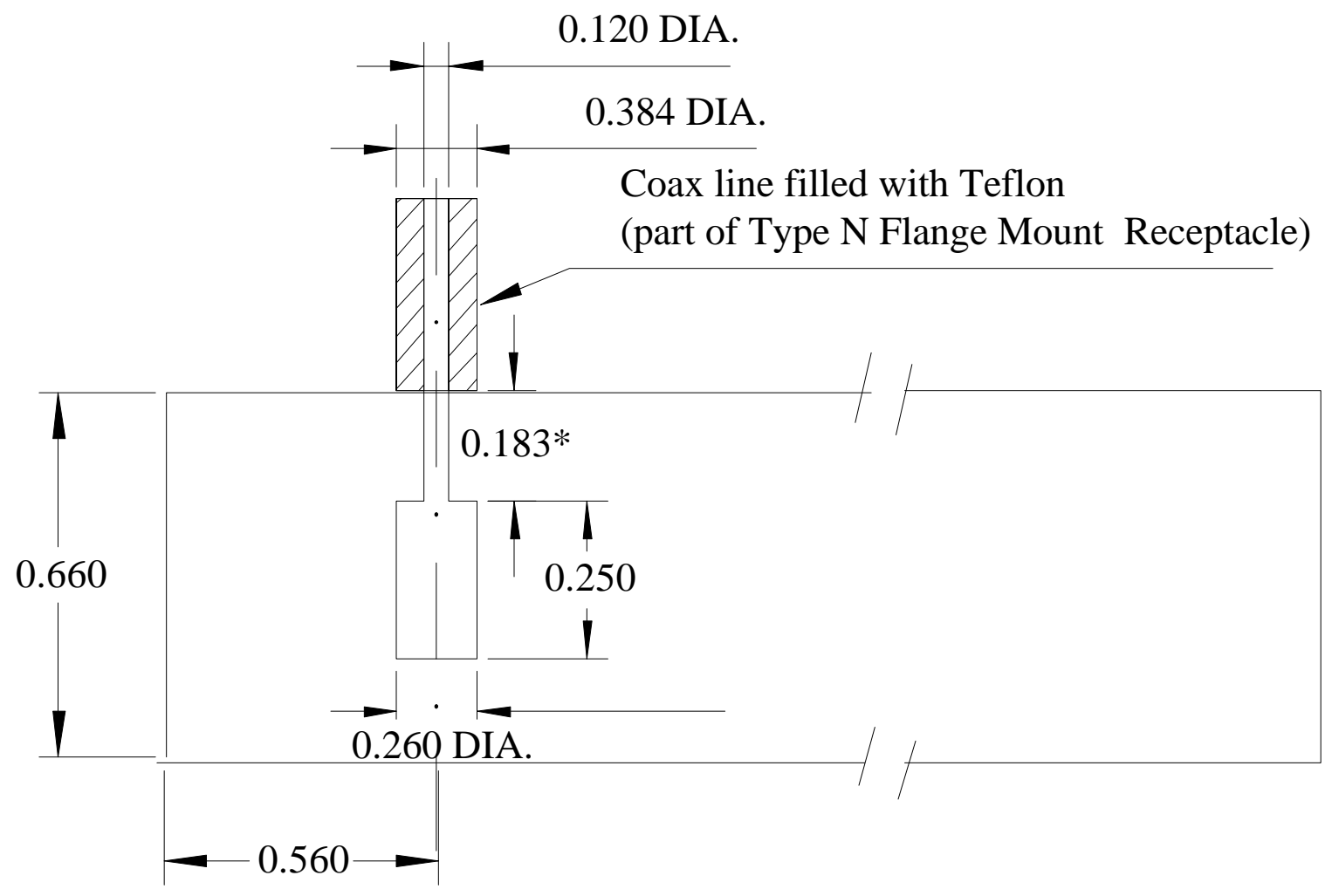

* 0.183 " was original design for perfect waveguide.

It was changed to $0.188 "-0.190 "$ when installed to the array.

Figure 5. Dimension of WG-Coax Launcher (Band 1 Kicker) 


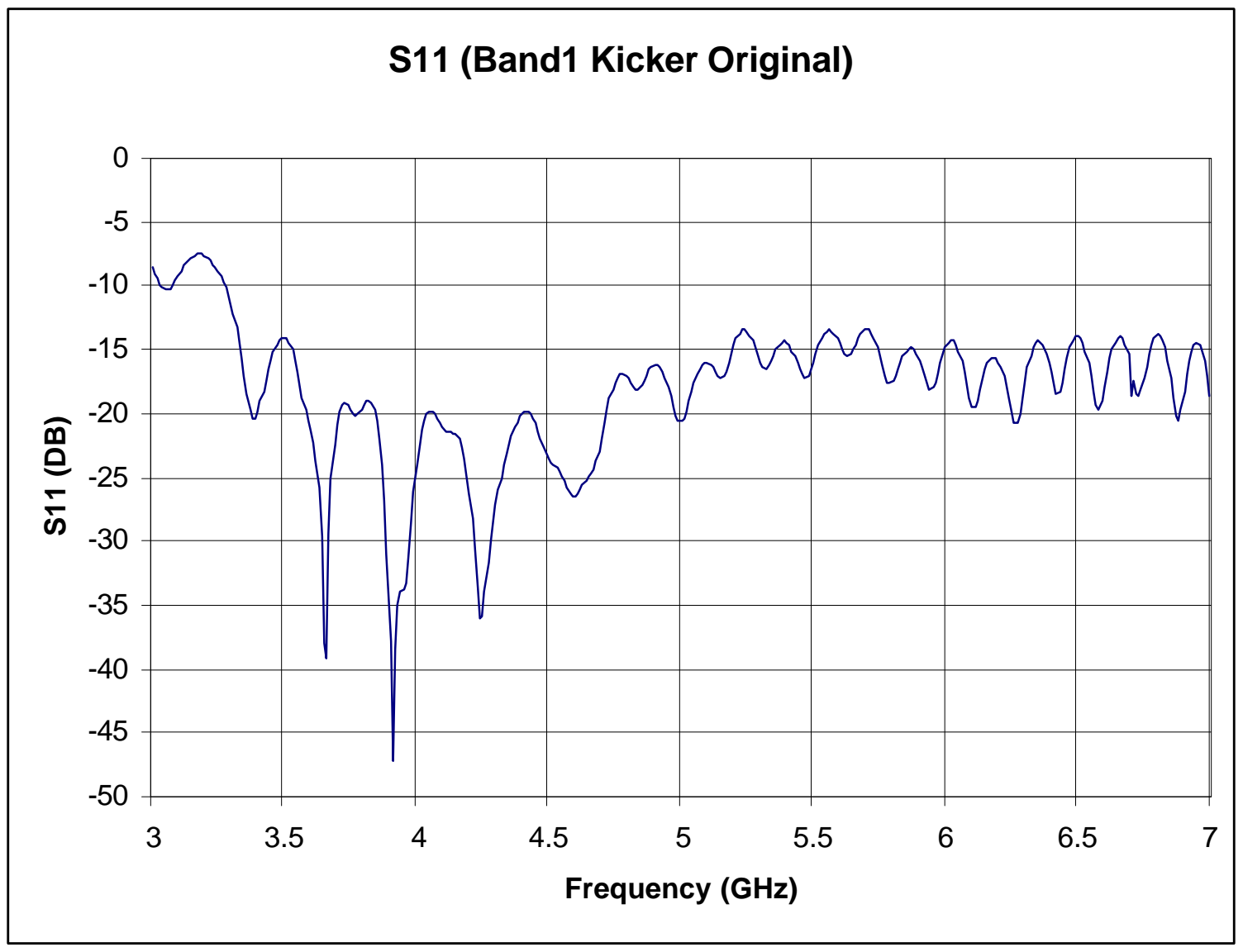

Figure 6. S11 of WG-Coax Launcher (Band 1, Kicker, measured using a test setup - a straight section of waveguide) 


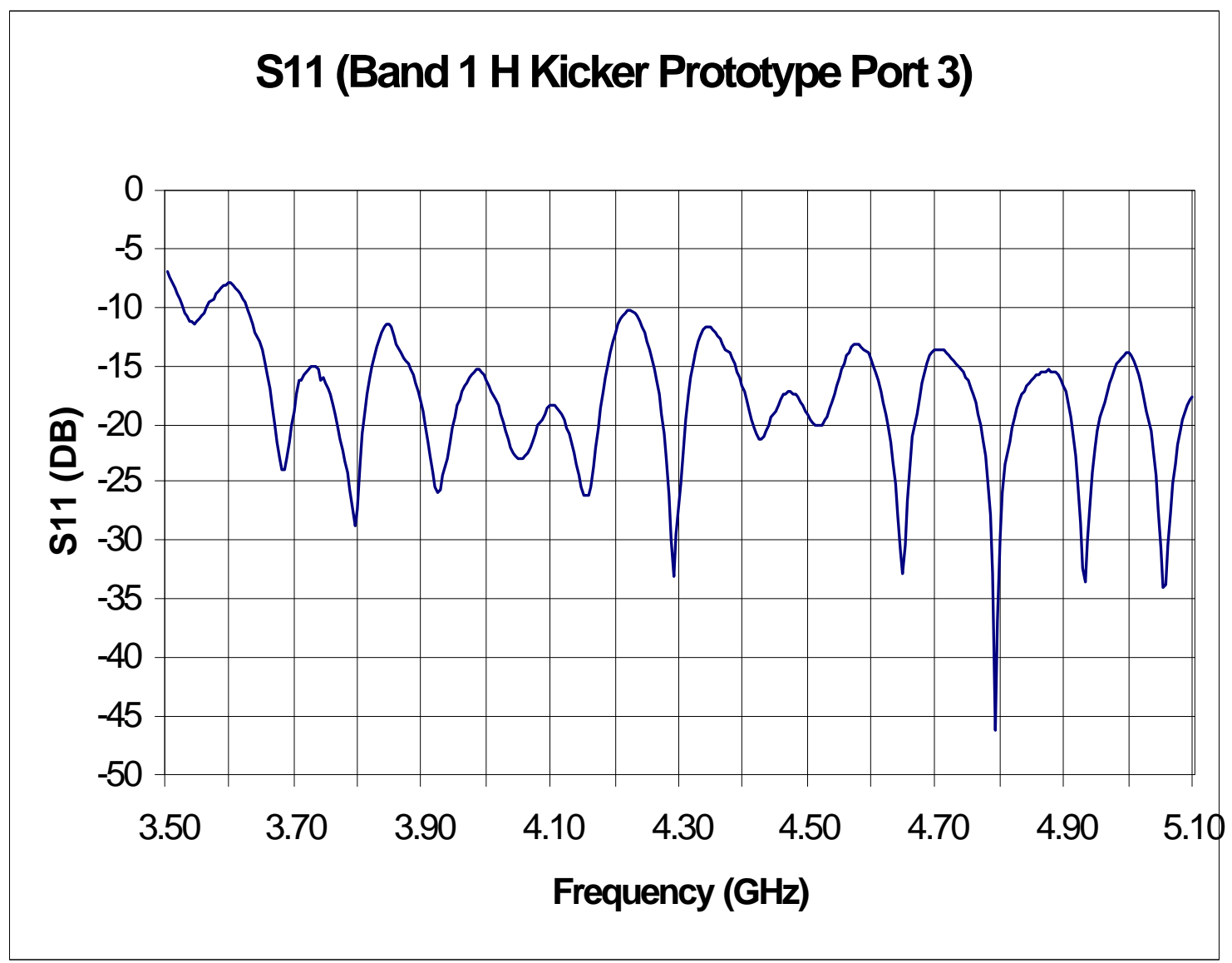

Figure 7. S11 of WG-Coax Launcher (Band 1, Kicker, measured after being installed onto prototype array) 


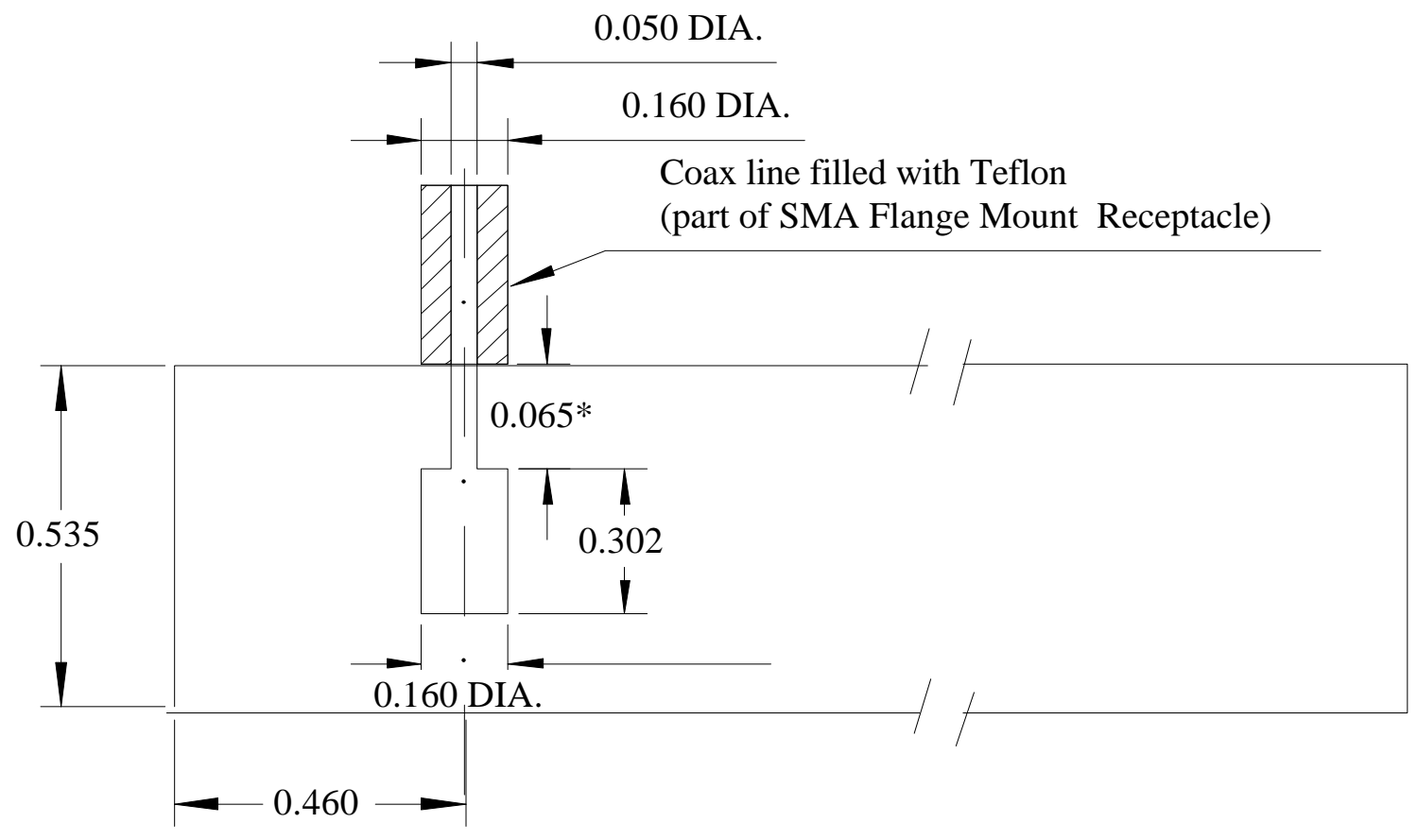

* 0.065" was the original design for straight SMA Flange Mount Receptacle. 0.075 " was used for elbow SMA Flange Mount Receptacle when installed to the array.

Figure 8. Dimension of WG-Coax Launcher (Band 2 Pickup) 


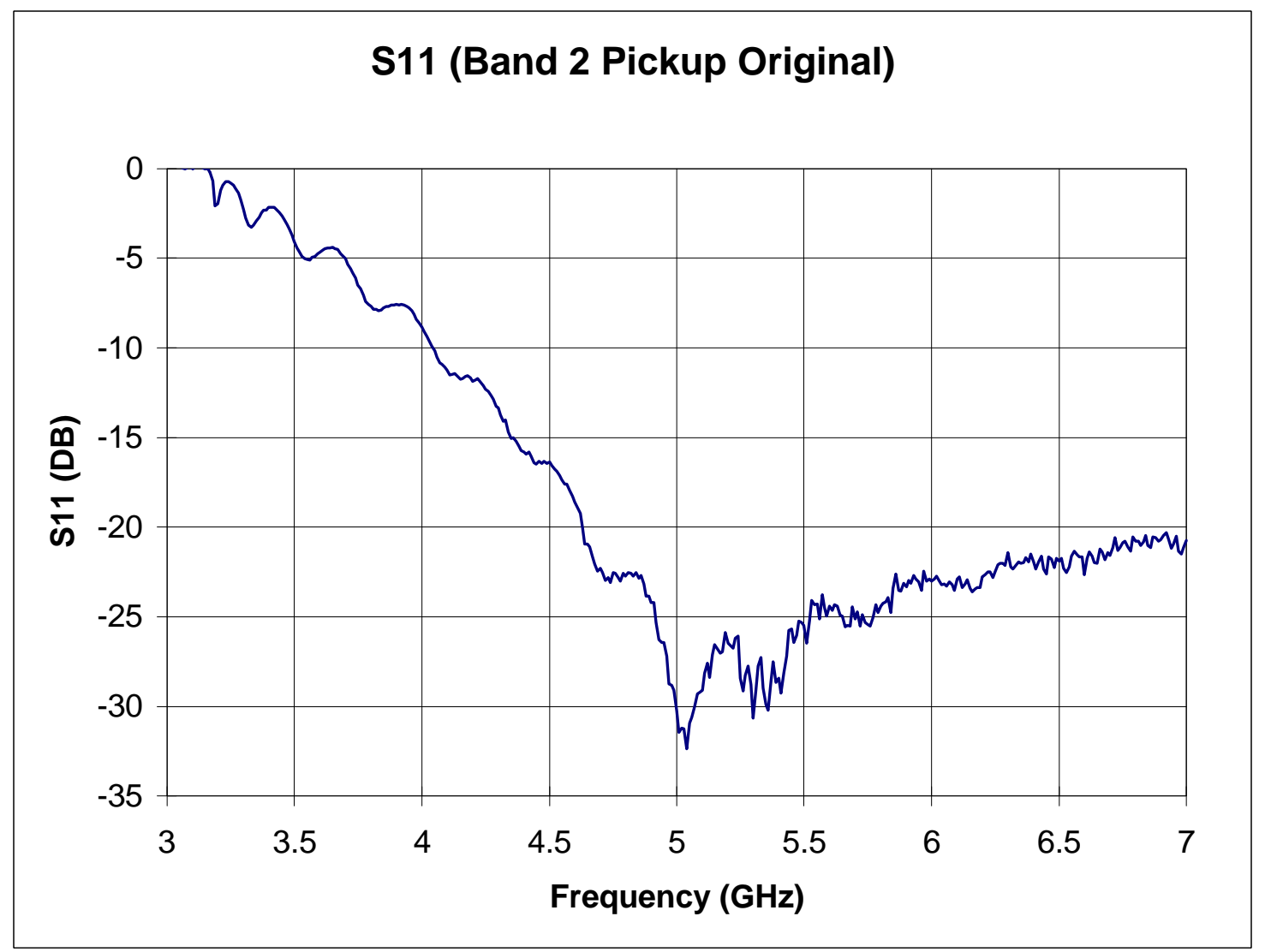

Figure 9. S11 of WG-Coax Launcher (Band 2, Pickup, measured using test setup- a straight section of waveguide) 


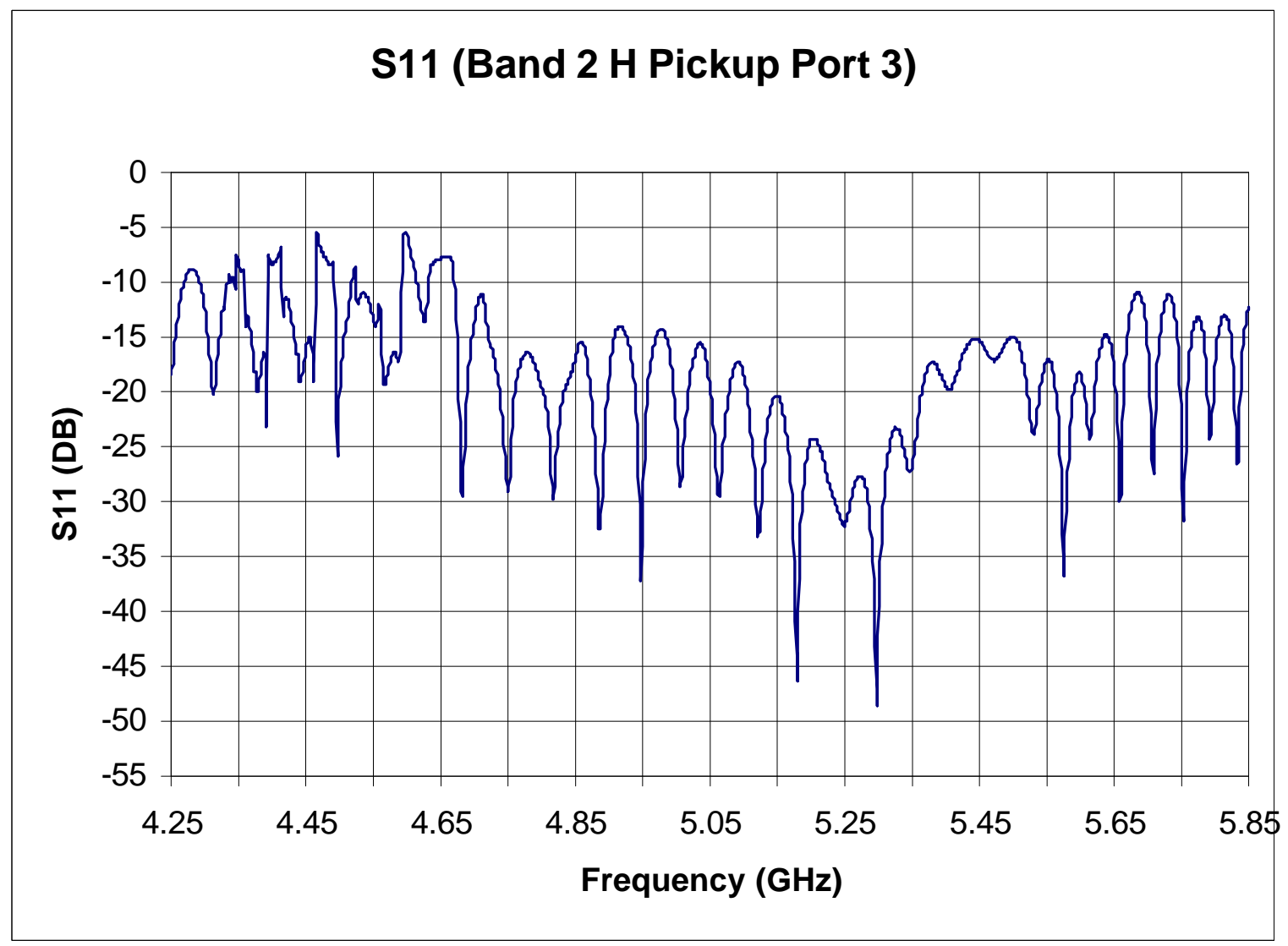

Figure 10. S11 of WG-Coax Launcher (Band 2, Pickup, upper stream, measured after being installed onto array) 


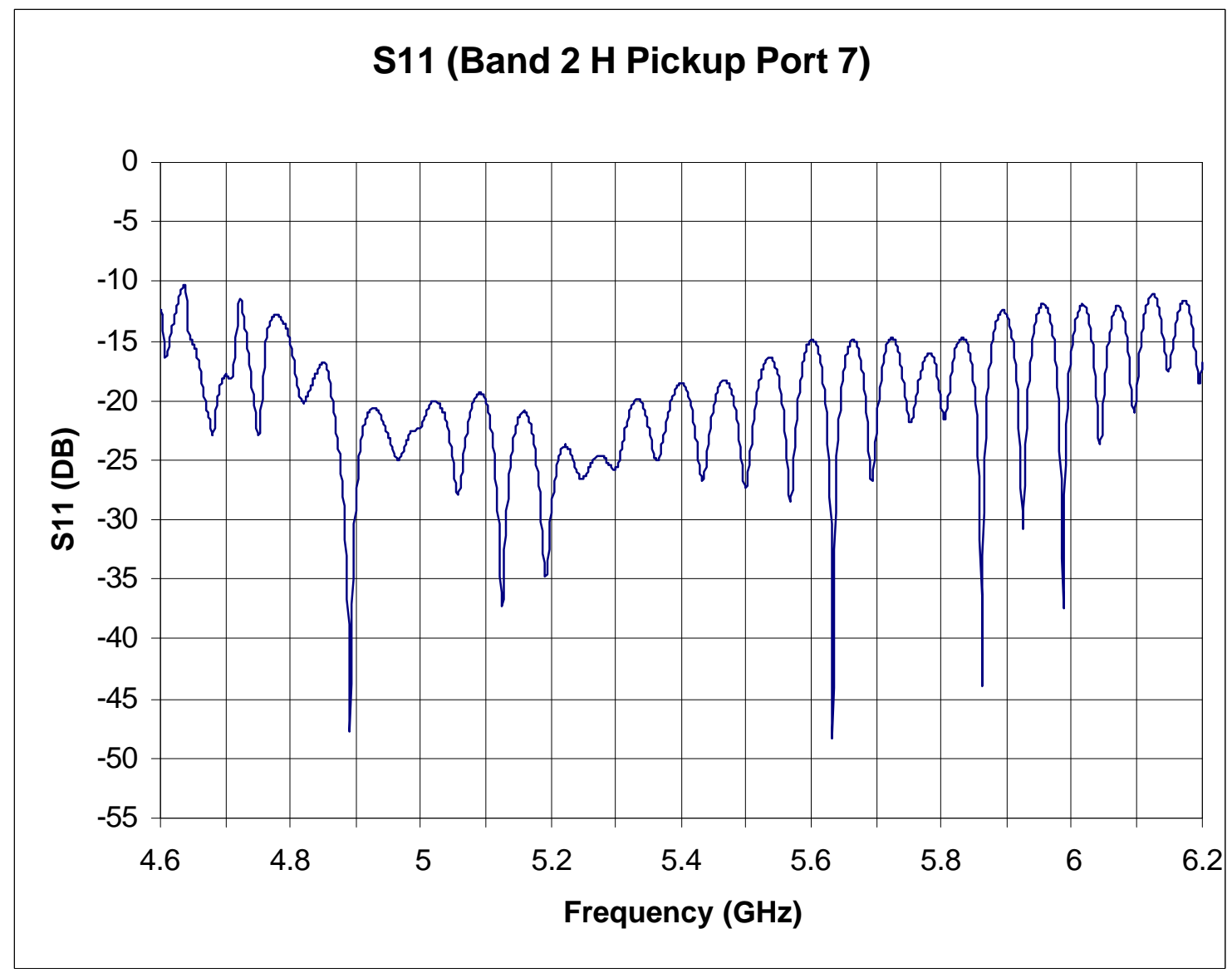

Figure 11. S11 of WG-Coax Launcher (Band 2, Pickup, down stream, measured after being installed onto array) 


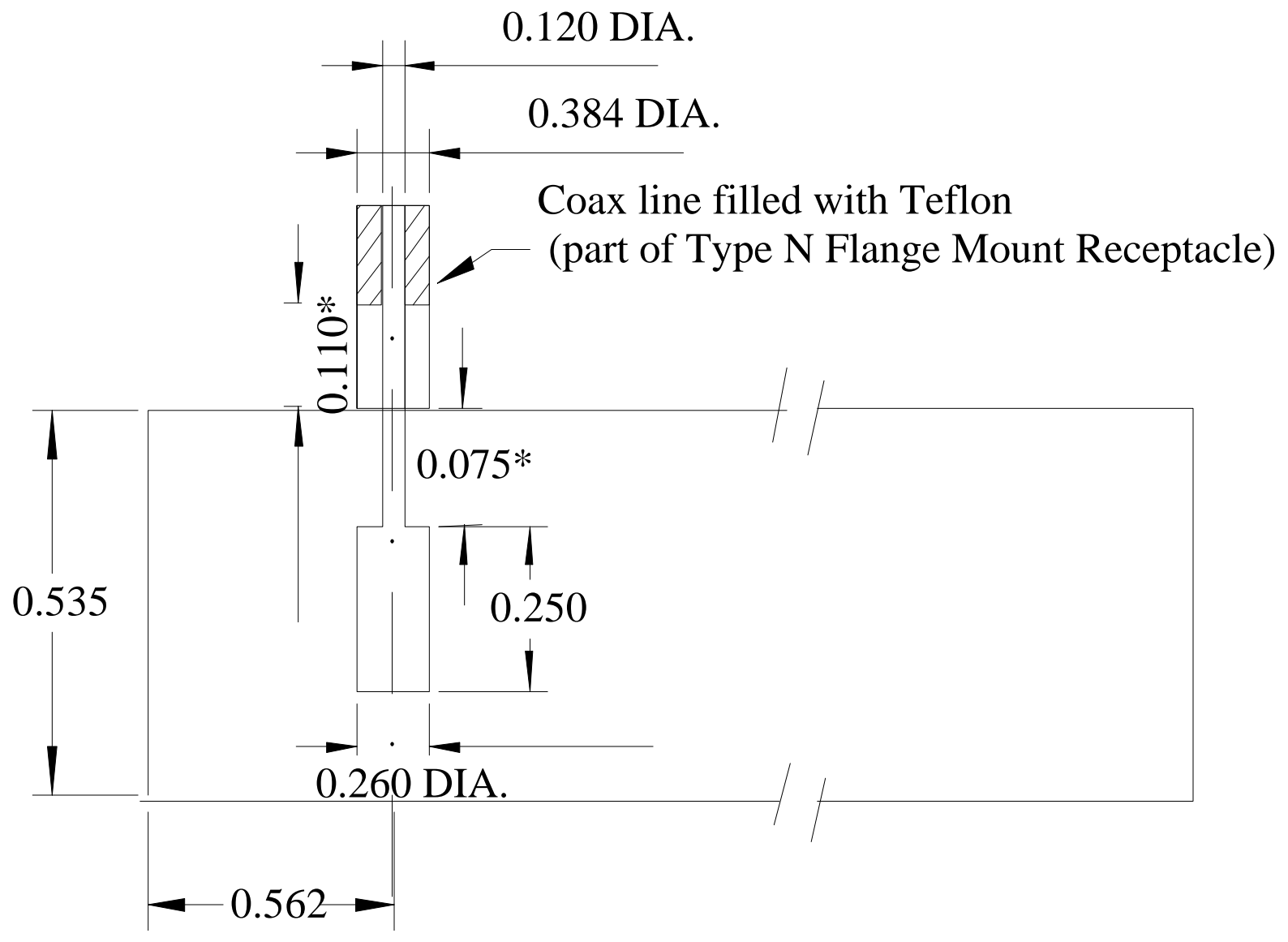

* $0.110 "$ was original design. It was changed to $0.084 "$ when install $0.075 "$ was original design, It was changed to $0.101 "$ when install

Figure 12. Dimension of WG-Coax Launcher (Band 2 Kicker) 


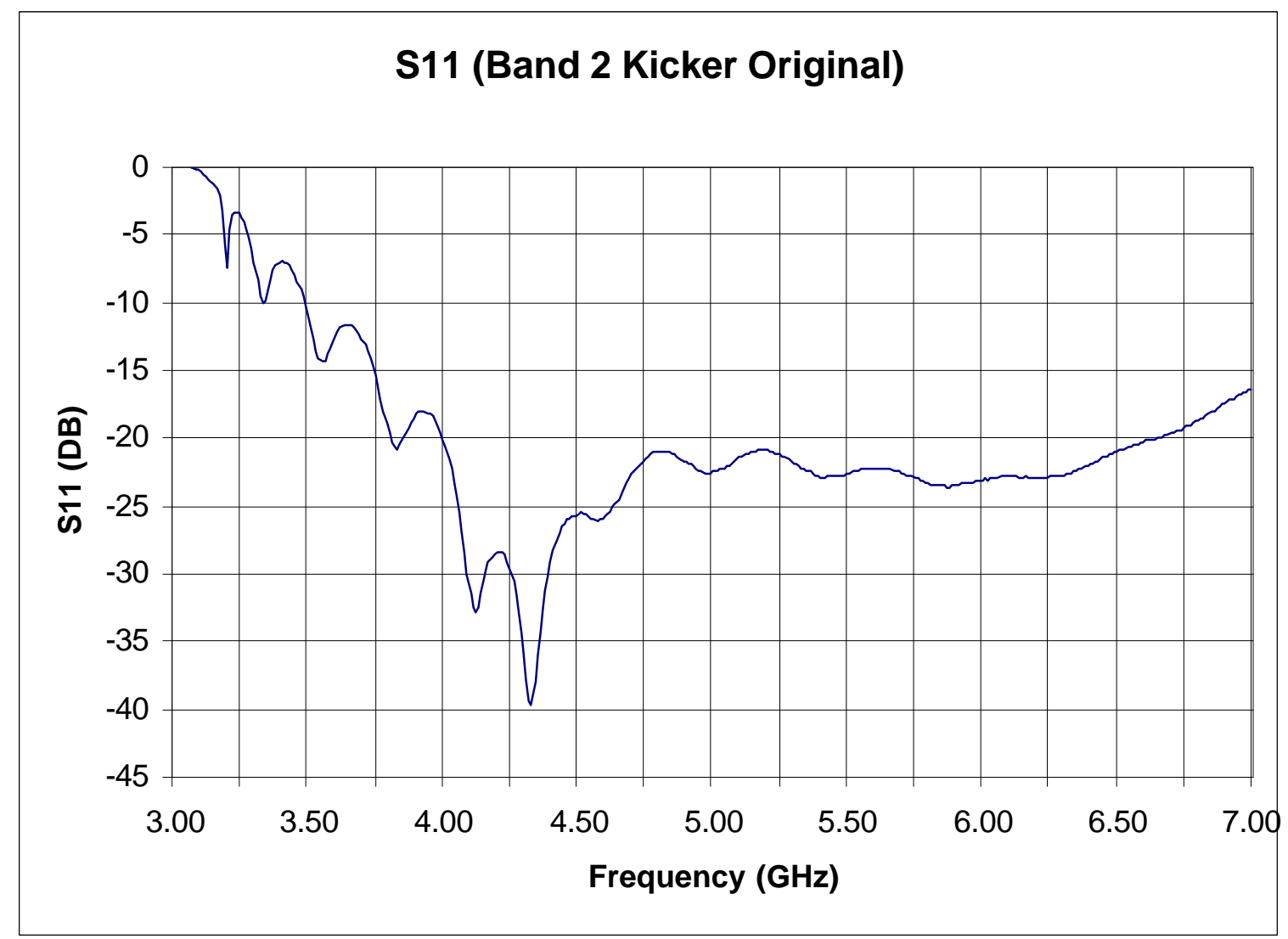

Figure 13. S11 of WG-Coax Launcher (Band 2, Kicker, measured using a test setup - a straight section of waveguide) 


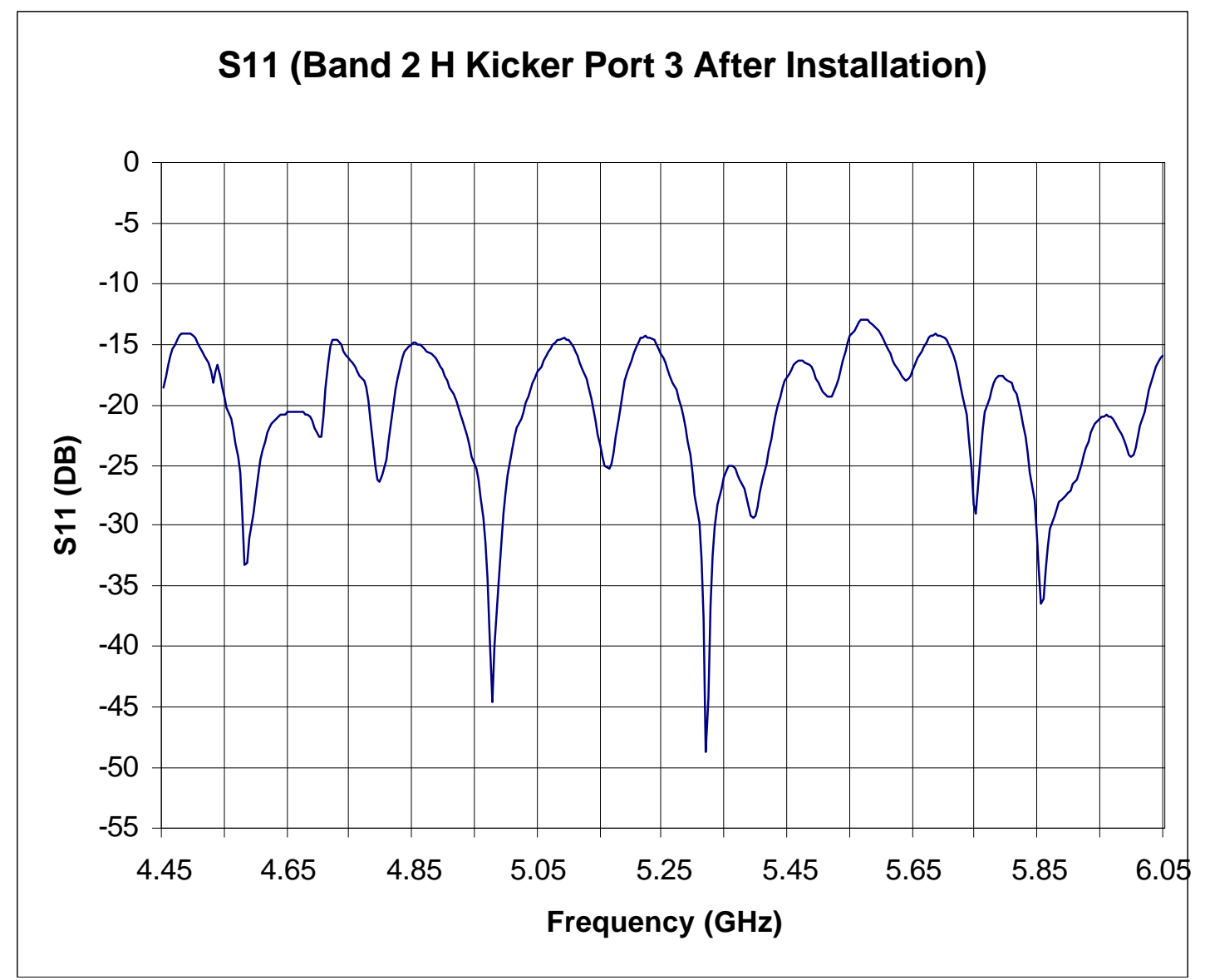

Figure 14. S11 of WG-Coax Launcher (Band 2, Kicker, measured after being installed onto array) 
